\title{
THE GROUP ALGEBRA OF A TORSION-FREE ONE-RELATOR GROUP CAN BE EMBEDDED IN A FIELD
}

\author{
BY JACQUES LEWIN ${ }^{1}$ AND TEKLA LEWIN
}

Communicated by Barbara L. Osofsky, May 5, 1975

Let $G_{1}, G_{2}$ be groups, $w$ a (skew) field, and $H$ a common subgroup of $G_{1}$ and $G_{2}$. Suppose that the group algebra $w G_{i}$ is embedded in a field $\bar{G}_{i}$ and suppose that the fields $\bar{H}_{1}$ and $\bar{H}_{2}$ generated by $w H$ in $\bar{G}_{1}$ and in $\bar{G}_{2}$ are $w H$-isomorphic. In order that the group algebra $w\left(G_{1}{ }_{H}^{*} G_{2}\right)$ be embedded in $\bar{G}_{1_{H_{1}}}{ }^{*} \bar{H}_{2} \bar{G}_{2}$ (* denotes coproduct ( $=$ free product with amalgamation) of groups and rings), it is sufficient that $\bar{H}_{i}$ and $w G_{i}$ be linearly disjoint over $w H$ in $\bar{G}_{i}$, i.e., that the multiplication map $\bar{H}_{i} \bigotimes_{W H} w G_{i} \rightarrow \bar{G}_{i}$ be injective.

If $R$ is a semifir, then [3, Chapter 7] there is a field $U(R)$, the universal field of fractions of $R$, embedding $R$ such that each automorphism of $R$ extends to an automorphism of $U(R)$. If $F$ is a free group, $w F$ is a semifir and has a universal field of fractions.

TheOREM 1. Let $G=\operatorname{gp}\langle t, x, y, \ldots, z ; R(t, x, y, \ldots, z)\rangle$ be a torsionfree one-relator group and $w$ a field. Then $w G$ can be embedded in a field $\bar{G}$ with the following property: If $S \subset\{t, x, y, \ldots, z\}$ and $S$ omits at least one letter involved in $R$, then the subfield $\overline{\mathrm{gp}(S)}$ of $\bar{G}$ generated by the free group algebra $w(\operatorname{gp}(S))$ is its universal field of fractions. Further, $\overline{\operatorname{gp}(S)}$ and $w G$ are linearly disjoint over $w(\mathrm{gp}(S))$ in $\bar{G}$.

SKETCH OF PROOF. Let the complexity of $R$ (which we assume is cyclically reduced) be the length of $R$ minus the number of letters involved in $R$. The proof is by induction on the complexity of $R$. (If $R$ has complexity zero, then $G$ is free and $\bar{G}=U(w G)$ has the required properties [5] .)

CASE 1. $R$ has exponent sum zero on some letter, say $t$. By the proof of the Freiheitssatz $[7, \S 4.4]$, the normal closure $N$ of $\{x, y, \ldots, z\}$ in $G$ is a tree product

$$
\cdots * N_{-1} A_{-1,0}^{*} N_{0} A_{0,1}^{*} N_{1} * \cdots
$$

where $N_{0}$ is a one-relator group whose defining relator is less complex than $R$, $A_{0,1}$ is a free group generated by a proper subset of the generators of $N_{0}$, and $16 \mathrm{~A} 60$.

AMS (MOS) subject classifications (1970). Primary 16A26, 20F05; Secondary 16A06,

Key words and phrases. One-relator groups, group algebras, zero-divisors in group-rings, global dimension of group-rings.

${ }^{1}$ This research was partially supported by NSF Research Grant 33050. 
$N_{i}=t^{-i} N_{0} t^{i}, A_{i, i+1}=t^{-i} A_{0,1} t^{i}$.

To prove the theorem, we copy this construction with fields. By induction, $w N_{0}$ is embedded in a field $\bar{N}_{0}$. Choosing copies $\bar{N}_{i}$ of $\bar{N}_{0}$ (with the isomorphism $\bar{N}_{0} \rightarrow \bar{N}_{i}$ extending $N_{0} \rightarrow N_{i}$ ) and defining $\overline{A_{i, i+1}}$ accordingly, we form the tree of rings

$$
\cdots * \bar{N}_{-1} \quad \bar{A}_{-1,0}^{*} \bar{N}_{0} \bar{A}_{0,1}^{*} \bar{N}_{1}^{*} \cdots \cdot
$$

The colimit $R$ of this system (= tree product) is [2] a semifir with a universal field of fractions $\bar{N}$ which we can show embeds $w N$.

We now extend, via the automorphism induced by conjugation by $t, \bar{N}$ to a field $\bar{G}$ which embeds $w G$.

To complete the proof of the exponent sum zero case, we show that each of the usual expressions of $N$ as a tree product induces a corresponding tree product structure inside $\bar{N}$.

CASE 2. Changing notation slightly, assume now that

$$
G=\operatorname{gp}\left\langle a, b, \ldots, z ; R^{\prime}(a, b, \ldots, z)\right\rangle
$$

and that $R^{\prime}$ has nonzero exponent sum on every letter. Let $H=G * \mathrm{gp}\langle t\rangle$. Two letters, say $a$ and $b$, and integers $k, l$ can be found such that if we set $x=a t^{k}$, $y=b t^{l}$, and $R(t, x, y, \ldots, z)=R^{\prime}\left(x t^{-k}, y t^{-l}, \ldots, z\right)$, then

$$
H=\mathrm{gp}\langle t, x, y, \ldots, z ; R(t, x, \ldots, z)\rangle
$$

is such that $R$ has exponent sum zero on $t$ and such that $N_{0}$, obtained as in Case 1 , has a defining relator less complex than $R^{\prime}$. We then work with $H$ and find that the subfield of $\bar{H}$ generated by $W G$ is a field with the required properties.

COROLlaRY. If $H$ is any group such that $w H$ can be embedded in a field $\bar{H}$, then $w(H \times G)$ can be embedded in a field (by choosing $w$ to be $\bar{H}$ in Theorem 1).

CoRollary. If $A$ is a commutative domain, then $A G$ is regular, i.e., $A G \otimes_{A} A G$ is a domain.

Now write $G=F / P$, where $F$ is a free group and $P$ is the normal closure of the element $R$. let $p$ be the augmentation ideal of $P$ in $A F$. From the universal derivation sequence,

$$
0 \rightarrow \mathfrak{p} / \mathfrak{p}^{2} \rightarrow T(A F ; A G, A G)=\Omega \rightarrow A G \otimes_{A} A G \rightarrow A G \rightarrow 0,
$$

[4], [1] we obtain

Theorem 2. If $A=\mathrm{Z}$, then $\mathrm{p} / \mathrm{p}^{2}$ is a free $\mathrm{ZG}$-bimodule (cf. [6]).

If $A$ is a PID, then the global dimension of $A G$ is at most 3.

If $A$ is a field, then the global dimension of $A G$ is at most 2. 
Proofs will appear in Communications in Algebra.

ADDED IN PROOF. The global dimension statements of Theorem 2 also follow from Corollary 4.2 of F. Waldhausen's $K$-theory of generalized free products (mimeographed).

\section{REFERENCES}

1. G. Bergman and W. Dicks, On universal derivations, J. Algebra (to appear).

2. P. M. Cohn, On the free product of associative rings. III, J. Algebra 8 (1968), 376-383; correction, ibid. 10 (1968), 123. MR 36 \#170; 37 \#121.

3. - Free rings and their relations, Academic Press, New York and London, 1971.

4. J. Lewin, A matrix representation for associative algebras. I, Trans. Amer. Math. Soc. 188 (1974), 293-308.

5. - Fields of fractions for group algebras of free groups, Trans. Amer. Math. Soc. 192 (1974), 339-346.

6. R. C. Lyndon, Cohomology theory of groups with a single defining relation, Ann. of Math. (2) 52 (1950), 650-665. MR 13, 819.

7. W. Magnus, A. Karrass and D. Solitar, Combinatorial group theory: Presentations of groups in terms of generators and relations, Pure and Appl. Math., Vol. 13, Interscience, New York and London, 1966. MR 34 \#7617.

DEPARTMENT OF MATHEMATICS, SYRACUSE UNIVERSITY, SYRACUSE, NEW YORK 13210 